\title{
EINIGE BEZEICHNUNGEN
}

\section{Vierdimensionale Bezeichnungen}

Vierdimensionale Tensorindizes werden mit griechischen Buchstaben $\lambda, \mu, \nu, \ldots$ bezeichnet und nehmen die Werte $0,1,2,3$ an.

Es wird die 4-Metrik mit der Signatur (+- - ) verwendet. Der metrische Tensor ist $g_{\mu \nu}\left(g_{00}=1, g_{11}=g_{22}=g_{33}=-1\right)$.

Die Komponenten eines 4-Vektors werden in folgender Weise angegeben: $a^{\mu} \equiv\left(a^{\mathbf{0}}, \boldsymbol{a}\right)$.

Zur Vereinfarhung der Schreibweise von Formeln wird der Index der Komponenten von 4-Vektoren häufig weggelassen. $\left.{ }^{1}\right)$ Skalarprodukte von 4-Vektoren werden dabei einfach als $(a b)$ oder $a b$ geschrieben: $a b=a_{\mu} b^{\mu}=a_{0} b_{0}-\boldsymbol{a} \boldsymbol{b}$.

Der vierdimensionale Ortsvektor ist $x^{\mu}=(t, \boldsymbol{r})$, das vierdimensionale Volumenelement ist $d^{4} x$.

Der Operator für die Differentiation nach dem 4-Koordinaten ist $\partial^{\mu}=\partial / \partial x^{\mu}$.

Der antisymmetrische 4-Einheitstensor ist $e^{\pi \mu \nu}$ mit $e^{0123}=-e_{0123}$ $=+1$.

Die vierdimensionale $\delta$-Funktion ist $\delta^{(4)}(a)=\delta\left(a_{0}\right) \delta(a)$.

\section{Dreidimensionale Bezeichnungen}

Für die dreidimensionalen Tensorindizes werden lateinische Buchstaben verwendet: $i, k, l, \ldots$; sie nehmen die Werte $x, y, z$ an.

Dreidimensionale Vektoren werden als halbfett-kursive Buchstaben gesetzt oder griechische Buchstaben mit Pfeil.

Das dreidimensionale Volumenelement ist $d^{3} x$.

\section{Operatoren}

Operatoren werden gewöhnlich steil gesetzt.

$\psi$-Operatoren werden mit halbfetten Buchstaben $\boldsymbol{\psi}$ bezeichnet.

Die Kommutatoren oder Antikommutatoren zweier Operatoren sind $\{\mathrm{f}, \mathrm{g}\}_{ \pm}=\mathrm{f} \mathrm{g} \pm \mathrm{g} \mathrm{f}$.

Der transponierte Operator ist $\tilde{\mathrm{f}}$.

1) Diese Schreibweise ist in der heutigen Literatur weit verbreitet. Dieser Kompromiß zwischen den Vorräten an Buchstaben und den Erfordernissen der Physik erfordert natürlich vom Leser besondere Aufmerksamkeit. 
Der adjungierte (hermitesch konjugierte) Operator ist $\mathrm{f}^{+}$.

Der Operator für die Ladungskonjugation ist C.1 )

Der Operator einer räumlichen Spiegelung ist P. ${ }^{1}$ )

Der Operator für die Zeitumkehr ist T. ${ }^{2}$ )

\section{Matrixelemente}

Das Matrixelement des Operators $\mathbf{F}$ für den Übergang aus dem Anfangszustand $i$ in den Endzustand $f$ ist $F_{f i}$ oder $\langle f|\mathbf{F}| i\rangle$.

Die Bezeichnung $|i\rangle$ wird als abstraktes Symbol für einen Zustand unabhängig von der konkreten Darstellung der zugehörigen Wellenfunktion verwendet, $\langle f|$ ist das Symbol für den Endzustand (,,konjugiert komplexen" Zustand). ${ }^{3}$ )

Dementsprechend werden mit $\langle s \mid r\rangle$ die Koeffizienten in der Entwicklung eines Systems von Zuständen mit den Quantenzahlen $r$ nach Zuständen mit den Quantenzahlen $s$ bezeichnet: $|r\rangle=\sum_{\delta}|s\rangle\langle s \mid r\rangle$.

Die reduzierten Matrixelemente der sphärischen Tensoren sind $\langle f|| F \| i\rangle$.

\section{Dirac-Gleichung}

Die Drrac-Matrizen sind $\gamma^{\mu}$ mit $\left(\gamma^{0}\right)^{2}=1,\left(\gamma^{1}\right)^{2}=\left(\gamma^{2}\right)^{2}=\left(\gamma^{3}\right)^{2}=$ $=-1$; ferner treten die Matrizen $\vec{\alpha}=\gamma^{0} \vec{\gamma}$ und $\beta=\gamma^{0}$ auf. Ausdrücke in Spinor- und in Standarddarstellung sind $(21,3),(21,16),(21,20)$ (S. 80, 83).

$$
\begin{aligned}
& \gamma^{5}=-i \gamma^{0} \gamma^{1} \gamma^{2} \gamma^{3},\left(\gamma^{5}\right)^{2}=1 \text { (s. }(22,18) \text { auf S. 88). } \\
& \sigma^{\mu \nu}=1 / 2\left(\gamma^{\mu} \gamma^{\nu}-\gamma^{\nu} \gamma^{\mu}\right) \text { (s. }(28,2) \text { auf S. 107). }
\end{aligned}
$$

Das Produkt eines 4-Vektors mit den Drrac-Matrizen ist $\hat{a}=(a \gamma)=$ $=a_{\mu} \gamma^{\mu}$.

Diracsche Konjugation: $\bar{\psi}=\psi^{*} \gamma^{0}$.

Die PAOLI-Matrizen $\vec{\sigma}=\left(\sigma_{x}, \sigma_{y}, \sigma_{z}\right)$ sind auf S. 79 definiert.

Die Indizes von $4-$ Spinoren werden mit $\alpha, \beta, \ldots$ und $\dot{\alpha}, \dot{\beta}, \ldots$ bezeichnet und nehmen die Werte 1,2 und $\dot{1}, \dot{2}$ an.

Die Bispinorindizes $i, k, l, \ldots$ nehmen die Werte $1,2,3,4$ an.

\section{Fourier-Entwicklung}

Die dreidimensionale Entwicklung ist

$$
f(\boldsymbol{r})=\int f(\boldsymbol{k}) e^{i \boldsymbol{k} \boldsymbol{r}} \frac{d^{3} k}{(2 \pi)^{3}}, \quad f(\boldsymbol{k})=\int f(\boldsymbol{r}) e^{-i \boldsymbol{k} \boldsymbol{r}} d^{3} x
$$

Entsprechendes gilt für den vierdimensionalen Fall.

1) Von den englischen Worten charge (Ladung) bzw. parity (Parität).

${ }^{2}$ ) Auch für das Symbol des zeitgeordneten Produkts wird der Buchstabe T verwendet.

3) Bezeichnung nach DIRac. 


\section{Maßeinheiten}

Wenn nicht besonders darauf hingewiesen wird, werden die relativistischen Einheiten $\hbar=1$ und $c=1$ verwendet. In diesen Einheiten ist das Quadrat der Elementarladung $e^{2}=1 / 137$.

Die atomaren Maßeinheiten sind: $e=1, \hbar=1, m=1$. In diesen Einheiten ist $c=137$. Atomare Längen-, Zeit- und Energieeinheit sind $\hbar^{2} / m e^{2}, \hbar^{3} / m e^{4}$ bzw. $m e^{4} / \hbar^{2}$ (die Größe Ry $=m e^{4} / 2 \hbar$ wird als Rydberg bezeichnet).

Die normalen Einheiten werden dem absoluten (GAUssschen) Maßsystem entnommen.

\section{Konstanten}

Lichtgeschwindigkeit $c=2,997925 \cdot 10^{10} \mathrm{~cm} / \mathrm{s}$.

Elementarladung $e=4,803: 10^{-10} \mathrm{cgs}-E i n h e i t e n$.

Elektronenmasse $m=9,110 \cdot 10^{-28} \mathrm{~g}$.

Plancksches Wirkungsquantum $\hbar=1,055 \cdot 10^{-27} \mathrm{erg} \cdot \mathrm{s}$.

Feinstrukturkonstante $\alpha=e^{2} / \hbar c ; 1 / \alpha=137,04$.

BoHRscher Radius $\hbar^{2} / m e^{2}=5,292 \cdot 10^{-9} \mathrm{~cm}$.

Klassischer Elektronenradius $r_{e}=e^{2} / m c^{2}=2,818 \cdot 10^{-13} \mathrm{~cm}$.

Compton-Wellenlänge eines Elektrons $\hbar / m c=3,862 \cdot 10^{-11} \mathrm{~cm}$.

- Ruhenergie eines Elektrons $m c^{2}=0,5110 \cdot 10^{6} \mathrm{eV}$.

Atomare Energieeinheit $m e^{4} / \hbar^{2}=4,360 \cdot 10^{-11} \mathrm{erg}=27,21 \mathrm{eV}$.

BoHRsches Magneton e $\hbar / 2 m c=9,274 \cdot 10^{-21} \mathrm{erg} / \mathrm{Gau}$.

Protonenmasse $m_{p}=1,673 \cdot 10^{-24} \mathrm{~g}$.

Compton-Wellenlänge eines Protons $\hbar / m_{p} c=2,103 \cdot 10^{-14} \mathrm{~cm}$.

Kernmagneton $e \hbar / 2 m_{p} c=5,051 \cdot 10^{-24} \mathrm{erg} / \mathrm{Gauß}$.

Hinweise auf andere Bände dieses Lehrbuches sind mit Ziffern versehen: I (Mechanik, 1967), II (Feldtheorie, 1970), III (Quantenmechanik, 1967). 
